dynamics indicate that Ceres would collide with one of the 30 asteroids capable of fragmenting it every $10^{10} \mathrm{yr}$ indicating that the large asteroids are original planetesimals. More- over, the dispersion of fragments after collision can produce orbits of reasonable inclination to the ecliptic just like those of the asteroids.

D.W.H.

\title{
Cell movements in Hydra
}

CELL movement is a fundamental component of morphogenesis. Cells may move as three-dimensional masses, in sheets or as individuals. The movements must be controlled by signals available to the cells, and the signals may be involved in the control of development in general. One can therefore deduce much about developmental control from observing cell movements; for example, melanocyte migration in amphibians is under chemotactic control, as is the aggregation of slime mold amoebae.

In Hydra, Wolpert has shown that much morphogenesis is possible by a combination of cell movement and differentiation without any increase in cell number. Herlands and Bode (see Nature, 248, 387; 1974) have now published observations on nematocyte migration, which confirm the general model for the control of Hydra development put forward by Wolpert and his collaborators.

Nematocytes are stinging cells used for the capture and paralysis of prey. They arise by differentiation from interstitial cells in Hydra's body column, and migrate into the tentacles where they position themselves in the ectoderm. Although there are at least four types of nematocyte, Herlands and Bode examined the two commonest, desmonemes and stenoteles.

The authors grafted the upper halves of Hydra labelled with ${ }^{3} \mathrm{H}$-thymidine to unlabelled lower halves, and also made the reverse grafts. They examined the originally unlabelled halves for labelled nematocytes. Nematocyte migration was strongly biased apically; indeed, basal migration only occurred towards buds. There was no migration into the peduncle.

As there was thus already a clear suggestion of apical preference in nematocyte migration, the authors tried further grafting experiments to determine whether the signal was produced by the head or was some underlying property of the cells through which the nematocytes migrate. The authors labelled gastric regions of $H$. attenuata with ${ }^{3} \mathrm{H}$-proline and grafted unlabelled heads to either the basal or apical ends. They then measured the rate of accumulation of labelled nematocytes in the unlabelled, grafted heads. They found that stenoteles accumulate at the same rate in basal and apical heads, but that desmonemes, for the first 3 days after grafting, accumulate in apical heads at a greater rate. After 3 days there was an increasing rate of migration by desmonemes into basal heads. These times correlate well with the times for polarity reversal by basally grafted heads found by earlier workers.

This interpretation was checked by grafting a head to the basal end and a peduncle to the apical end of a labelled gastric region. The addition of a peduncle is known to accelerate polarity reversal, and indeed there was no indication that the desmonemes migrated according to the original tissue polarity. Stenoteles, as before, migrated to the head regardless of polarity.

These results show that two kinds of signal are usedboth an underlying property of the tissue, which may correspond to Wolpert's "positional value" and which can determine polarity, and a signal emanating from the head independent of polarity. This might be a chemotactic signal. The signals closely correspond to those already invoked for models of the control of Hydra development.

From a Correspondent

\section{An SV40 that doesn't help adenovirus}

THE inability of human adenoviruses to undergo a productive cycle of growth in simian cells-possibly because of the failure of a late function-can be overcome by coinfection with simian virus 40 (SV40). Adenovirus T-antigen (Feldman et al., J. Bact., 91, 813; 1966; Malmgren et. al., ibid., 262) and viral DNA are produced (Reich et al., Proc. natn. Acad. Sci. U.S.A., 55, 336; 1966; Baum et al., Virology, 34, 373; 1966), whereas the production of some late protein is inhibited in monkey cells (Friedman et al., J. Virol., 5, 586; 1970; Henry et al., Nature new Biol., 233, 39; 1971). The region of SV40 DNA that specifies its helper function was located in an adeno-SV40-nondefective hybrid by Lewis and his colleagues (Proc. natn. Acad. Sci. U.S.A., 63, 1128; J. Virol., 11, 655; 1971). These workers selected, from adenovirus populations that had been grown in the presence of SV40, mutants that were able to grow productively on monkey cells without the assistance of exogenous SV40 virus.

Some of these mutants contained segments of SV40 DNA covalently linked to adenovirus DNA sequences. In these adeno-SV40 hybrids, the region of the adenovirus substituted for by the SV40 sequences does not seem to be essential for adenovirus growth. The substitutions of SV40 sequences all begin at the same place in the adenovirus genome ( $14 \%$ from one end) but in different mutants the SV40 sequences can range in amount from $11-43 \%$ of the SV40 genome, the smallest being $17 \%$ of the genome (see $J$. Virol., 12, 643; 1973). All these SV40 sequences start from one place on the SV40 physical map which is 0.11 map units to one side of the $E_{c o} \mathrm{R}_{1}$ cleavage site (see J. Virol., 12, 653;
1973). The exact nature of the SV40 helper function is not known but it has been mapped in a region of the genome that is thought to specify early functions.

Jerkofsky and Rapp tested the enhancing ability of some temperature sensitive mutants of SV40. Both late mutants, which did synthesise viral DNA, and an early mutant, $t s$ A7, which did not synthesise viral DNA, but did induce host DNA synthesis (J. Virol., 8, 516; 1971), were able to enhance the production of adenovirus at the nonpermissive temperature in monkey cells. These results support their idea (Virology, 51, 466; 1973) that an early function of SV40, possibly related to the induction of host DNA synthesis, is involved.

Kimura, in this issue of Nature, has tested the helping ability of temperature sensitive mutants of three complementation groups defined by Kimura and Dulbecco (Virology, 52, 529; 1973). He also finds that late mutants will enhance adenovirus growth at the nonpermissive terr:perature. The early mutant ts 640 was unable to facilitate adenovirus growth at the nonpermissive temperature although enhancement was normal at the permissive temperature. This mutant $t s 640$, like $t s \mathrm{~A} 7$, is temperature sensitive for the production of viral DNA. It is not known whether $t s 640$ induces host DNA synthesis; it may differ in this respect and thus represent a second class of early mutants of SV40 (possibly a fourth complementation group). A further characterisation of $t s 640$ should be accomplished soon.

Kimura has mapped his genetic marker in the SV40 helper function: it occurs between 0.11 and 0.28 map units on the SV40 physical map. From a Correspondent 